\title{
Diseño del biodigestor tipo laguna cubierta para el Establo de la Universidad Nacional Toribio Rodríguez de Mendoza, Chachapoyas, Amazonas
}

\section{Design of the covered lagoon biodigester for the Stable of the Toribio Rodríguez de Mendoza National University, Chachapoyas, Amazonas}

Miguel Ángel Barrena Gurbillón ${ }^{1^{*}} \mathbb{D}$, Perci Salazar Salazar ${ }^{1}$, Wildor Gosgot Ángeles ${ }^{1} \mathbb{D}$, Carla María Ordinola Ramírez $\mathbb{D}^{1}$, Milton Huanes Mariños

\section{RESUMEN}

Se diseñó el sistema de tratamiento de efluentes del Establo de la Universidad Nacional Toribio Rodríguez de Mendoza de Amazonas (UNTRM), para procesar estiércol de ganado vacuno. El biodigestor tipo laguna cubierta (BTLC) de $170 \mathrm{~m}^{3}$, debe ser excavado en el suelo e impermeabilizado con geomembrana, para procesar el estiércol de 40 vacas que producen $600 \mathrm{~kg}$ de estiércol/día, en $126 \mathrm{~m}^{3}$ de volumen de trabajo, con tiempo de retención hidráulico de 35 días. El BTLC tiene forma de tronco de pirámide invertida, de $3 \mathrm{~m}$ de profundidad y talud de $45^{\circ}$. La cúpula del biodigestor tiene $1,8 \mathrm{~m}$ de alto y $12 \times 9 \mathrm{~m}$ en su base (borde superior del BTLC), almacenará $133,56 \mathrm{~m}^{3} \mathrm{de}$ biogas. El volumen libre en el BTLC es de $44 \mathrm{~m}^{3}$; la capacidad de almacenamiento del sistema será de $177,56 \mathrm{~m}^{3}$, que equivale a 4,03 días de producción de biogas. La laguna para biol tiene forma de tronco de pirámide invertida impermeabilizada con geomembrana de $51,6 \mathrm{~m}^{3}$, de $1,5 \mathrm{~m}$ de profundidad y talud de $45^{\circ}$. La laguna para almacenar lodos de fondo tiene forma de tronco de pirámide invertida, de $113,39 \mathrm{~m}^{3}$, de $2,0 \mathrm{~m}$ de profundidad, talud de $45^{\circ}$, excavada en el suelo y reforzada con canto rodado y concreto. El biogas producido se empleará como combustible ecológico. Los bioabonos servirán para mejorar los suelos e incrementar la producción de los pastos para el ganado. La operación del BTLC contribuirá a la sostenibilidad de la actividad del establo.

Palabras claves: biogás, energías renovables, biodigestor tipo laguna cubierta.

\begin{abstract}
The effluent treatment system of the Stable of the Toribio Rodríguez de Mendoza National University of Amazonas (UNTRM) was designed to process cattle manure. The covered lagoon type biodigestor (BTLC) of $170 \mathrm{~m}^{3}$, must be excavated in the soil and waterproofed with geomembrane, to process the manure of 40 cows that produce $600 \mathrm{~kg}$ of manure/day, in $126 \mathrm{~m}^{3}$ of work volume, with time of hydraulic retention of 35 days. The BTLC has the shape of an inverted pyramid trunk, $3 \mathrm{~m}$ deep and $45^{\circ}$ slope. The dome of the biodigester is $1,8 \mathrm{~m}$ high and $12 \times 9 \mathrm{~m}$ at its base (upper edge of the BTLC), it will store $133,56 \mathrm{~m}^{3}$ of biogas. The free volume in the BTLC is $44 \mathrm{~m}^{3}$; the storage capacity of the system will be $177,56 \mathrm{~m}^{3}$, equivalent to 4,03 days of biogas production. The lagoon for biol has the shape of an inverted pyramid trunk of $51,6 \mathrm{~m}^{3}$, waterproofed with geomembrane, $1,5 \mathrm{~m}$ deep and $45^{\circ}$ slope. The lagoon for storing bottom mud has the shape of an inverted pyramid trunk, 113,39 $\mathrm{m}^{3}, 2,0 \mathrm{~m}$ deep, $45^{\circ}$ slope, excavated in the ground and reinforced with boulder and concrete. The biogas produced will be used as an ecological fuel. The bioabonos will serve to improve the soils and increase the production of pastures for livestock. The operation of the BTLC will contribute to the sustainability of the activity of the stable.
\end{abstract}

Keywords: biogas, renewable energies, covered lagoon type biodigestor.

\footnotetext{
${ }^{1}$ Instituto de Investigación para el Desarrollo Sustentable de Ceja de Selva, Universidad Nacional Toribio Rodríguez de Mendoza de Amazonas (UNTRM-A), Calle Higos Urco N³42-350-356, Calle Universitaria $\mathrm{N}^{\circ} 304$, Chachapoyas, Perú

${ }^{2}$ Universidad Privada Antenor Orrego (UPAO), Av. América Sur n ${ }^{3}$ 145, Urb. Monserrate, Trujillo, Perú

Autor de Correspondencia, e-mailmiguel.barrena@untrm.edu.pe
} 


\section{I.INTRODUCCIÓN}

El biodigestor tipo laguna cubierta (BTLC), es una poza excavada en el suelo con forma de tronco de pirámide invertida, con todo su interior recubierto con material plástico impermeable como la geomembrana de PVC o de polietileno; con el que también se tapa la poza conformando la cúpula para generar la condición anaeróbica. La mezcla estiércol:agua ingresa al BTLC por gravedad o bombeo, donde será procesada anaeróbicamente por alrededor de 40 días. El biogas, rico en metano, es almacenado en la parte superior (cúpula) del biodigestor, de donde es distribuido por tuberías hacia su uso final como combustible para calefacción o de un grupo electrógeno. El exceso de biogas puede quemarse en una antorcha, produciendo dióxido de carbono y agua; este $\mathrm{CO}_{2}$ en comparación al metano, es prácticamente inocuo, pues tiene 21 veces menos poder de gas de efecto invernadero, con lo que se disminuye el impacto ambiental del proceso productivo (Moncayo, 2014).

Según Nennich et al. (2005), una vaca en producción hace alrededor de 75,2 $\mathrm{kg}$ de estiércol/día, una vaca seca $38,6 \mathrm{~kg} /$ día, una vaquilla $24,5 \mathrm{~kg}$ /día y un becerro 12,4 kg/día. Sin embargo, DEC (2006), presenta un valor de $50 \mathrm{~L} / \mathrm{vaca} /$ día con un rango de 30-100 $\mathrm{L} /$ vaca/día ya que las cantidades de estiércol varían de acuerdo al peso del animal y las condiciones de crianza. Laines y Sosa (2013), reportan un biodigestor anaerobio tipo laguna cubierta de $67 \mathrm{~m}^{3}$, de geomembrana de PVC, con tubería de PVC para conducción de biogas y recirculación de biol, cargado con mezcla rumen de bovino:agua en proporción 4:1. Se agitó hidráulicamente con una bomba autocebante.

Se debe implementar tecnologías para procesar el estiércol en biodigestores anaeróbicos para generar biogas y abonos orgánicos que reducen las emisiones atmosféricas y los costos de fertilización en la producción de forrajes y el consumo energético en los establos, lo que a su vez contribuye a la disminución de emisiones de óxido nitroso de los fertilizantes y dióxido de carbono de los combustibles fósiles para la generación de energía. La implementación de mejores prácticas incrementa la productividad de leche por vientre y peso en animales para producción de carne, haciendo económicamente viable la implementación de tecnologías para reducir la huella de carbono (Bonilla y Lemus, 2012).

El diseño e instalación del sistema de tratamiento de efluentes del establo de la Universidad Nacional Toribio Rodríguez de Mendoza de Amazonas (UNTRM), permitirá procesar el estiércol del ganado vacuno en el biodigestor tipo laguna cubierta, en cuya cúpula se almacenará el biogas producido, que se empleará como combustible para cocina, lámpara de camiseta y motor de combustión interna. El biol y el biosol producidos, se emplearán como fertilizantes orgánicos para incrementar la producción de los pastos, con lo cual se estará realizando un reciclaje de nutrientes. Los bioabonos también se podrán comercializar.

\section{MATERIAL Y MÉTODOS}

En el Establo de la Universidad Nacional Toribio Rodríguez de Mendoza de Amazonas (UNTRM) se cría 40 vacas raza Jersey de pedrigee, por lo que se ha diseñado un sistema para procesar el estiércol y reducir su impacto ambiental produciendo biogas y bioabonos (biol y biosol). Este sistema consta de: sedimentador, biodigestor tipo laguna cubierta, laguna para biol, cámara para secado de lodos de fondo y un sistema de purificación y control de biogás. Para el diseño e implementación se consideró las siguientes variables:

- Tipo de suelo: Las lagunas se excavarán en suelo tipo arcillas expansivas que serán impermeabilizadas con geomembrana.

- Talud: Se empleó para el diseño un talud de $45^{\circ}$ debido a que el suelo es de tipo arcillas expansivas, con una compactación del 90\% Proctor para garantizar que no exista ningún tipo de protuberancias, que puedan dañar la geomembrana.

- Corona del biodigestor: El ancho de la corona, tendrá $3 \mathrm{~m}$ (libre de tuberías, registros, salida de gas, etc.).Se consideró una zanja de $0,25 \mathrm{~m}$ de profundidad x $0,15 \mathrm{~m}$ de ancho, para la viga de 
anclaje de la geomembrana de la cámara y de la cúpula del BTLC.

- Profundidad: La profundidad mínima de líquido en la laguna cubierta debe ser de $3,0 \mathrm{~m}$.

- Tiempo de retención hidráulica y dimensionamiento de la laguna cubierta: De la instalación y operación de biodigestores en diversos lugares, Barrena et al. (2013), propusieron la ecuación: $\mathrm{TRH}=-44,705 \ln (\mathrm{T})+160,394$; que modela el TRH en días para la producción de biogas en función de la temperatura $\left(\mathrm{T}:{ }^{\circ} \mathrm{C}\right)$ ambiente, con $\mathrm{R}^{2}=$ 0,924 .

- Volumen del biodigestor: Es igual al volumen del material a degradar, multiplicado por el TRH, más un volumen adicional para el almacenamiento de biogas (Barrena et al., 2013, SEMARNAT et al., 2010).

- Material de la cobertura: Se utilizará geomembrana de 1,0 $\mathrm{mm}$ de espesor para cubrir la poza del BTLC y taparlo para conformar su cúpula.

\section{RESULTADOS}

\section{Diseño del biodigestor tipo laguna cubierta (BTLC)}

Diseño de la cámara del BTLC

Para el diseño del BTLC se consideró a las 40 vacas lecheras de la raza Jersey de pedigree, del Establo de la UNTRM, de un peso promedio de $550 \mathrm{~kg}$, cada una evacúa aproximadamente $15 \mathrm{~kg}$ de estiércol por día, lo que hace un total de $600 \mathrm{~kg}$ de estiércol/día. La proporción estiércol:agua más adecuada es de 1:5, para tener mayor fluidez en el interior del biodigestor (Barrena et al., 2013), lo que hace 6 partes en total, por lo que se tendrá $3,6 \mathrm{~m}^{3}$ de mezcla estiércol:agua/día. Se consideró que el TRH en Chachapoyas es de 35 días, por lo que la cámara de fermentación del BTLC debe tener un volumen útil o de trabajo $(\mathrm{Vt})$ de $126 \mathrm{~m}^{3}$ que representará el 80\% del volumen de la cámara $(\mathrm{Vc})$, porque se debe dejar libre el $20 \%$ del volumen para que se acumule el biogás, entonces, el Vc es igual a 157,5 m³. Para conservar el calor en el BTLC, su profundidad será de 3,0 m (CNA, 2007). Se consideró que la cámara del biodigestor tenga la forma de un tronco de pirá- mide invertida, cuyo volumen (Vc) se calcula con la ecuación:

$$
V c=\frac{h}{3}(A B+A b+\sqrt{A B \times A b})
$$

Donde:

$\mathrm{AB}$ : área de base mayor $\left(\mathrm{m}^{2}\right)$; $\mathrm{Ab}$ : área de base menor $\left(\mathrm{m}^{2}\right)$; h: profundidad de la cámara $(\mathrm{m})$

Según la EPA(2001), AB = 2Ab

De la ecuación (1): $\mathrm{Ab}=35,68 \mathrm{~m}^{2}$

Si $1=5 a$, entonces, $\mathrm{a}=2,67 \mathrm{~m}$ y $\mathrm{l}=13,35 \mathrm{~m}$

$\mathrm{Si} \mathrm{L}=5 \mathrm{~A}$, entonces, $\mathrm{A}=3,78 \mathrm{~m}$ y $\mathrm{L}=18,9 \mathrm{~m}$

Las dimensiones de la cámara del biodigestor serán las siguientes (Figura 1): Base superior: $\operatorname{largo}=12 \mathrm{~m} \mathrm{y}$ ancho $=9 \mathrm{~m}$; base inferior: $\operatorname{largo}=6 \mathrm{~m}$ y ancho $=3 \mathrm{~m}$; profundidad $=3 \mathrm{~m}$; talud $=45^{\circ} \mathrm{y}$ volumen $=170 \mathrm{~m}^{3}$
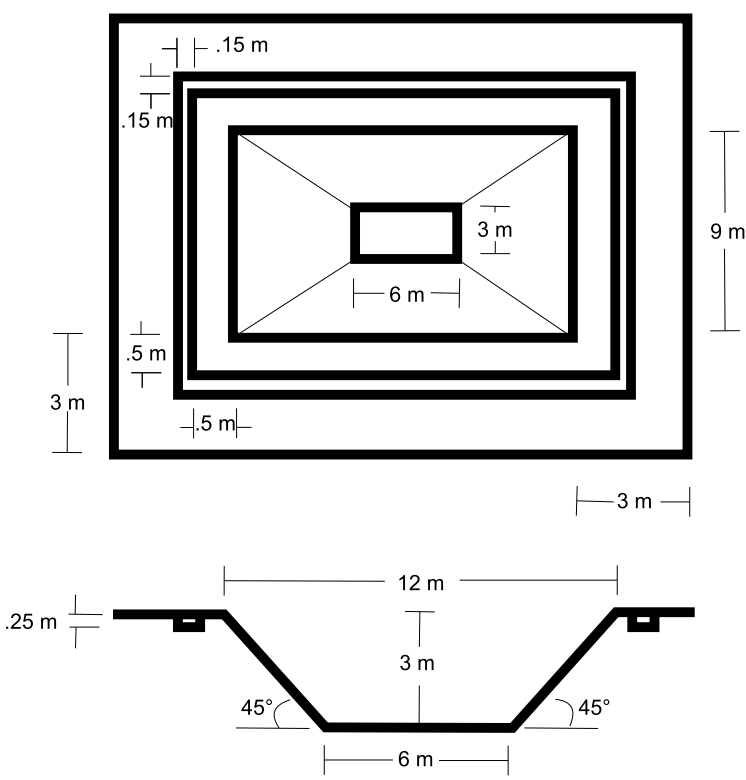

Figura 1. Vista superior y lateral de la cámara del biodigestor.

\section{Diseño de la cúpula del BTLC}

La cúpula del biodigestor servirá para almacenar el biogás producido. La cúpula se inflará hasta $1,8 \mathrm{~m}$ en su parte central. Para el cálculo de sus dimensiones se tuvo en cuenta la Figura 2, donde:

Lado del polígono inscrito $(X Y): 1 p=9 \mathrm{~m}$

Sagita (WT): $\mathrm{S}=1,8 \mathrm{~m}$; Radio: $\mathrm{r}=\left(1 \mathrm{p}^{2}+4 \mathrm{~S}^{2}\right) / 8 \mathrm{~S} ; \mathrm{r}=$ $6,53 \mathrm{~m} ; \operatorname{sen}=(\operatorname{lp} / 2) / \mathrm{r} ; \alpha=43,56^{\circ} ; \theta=2 \alpha ; \theta=87,12^{\circ}$. 


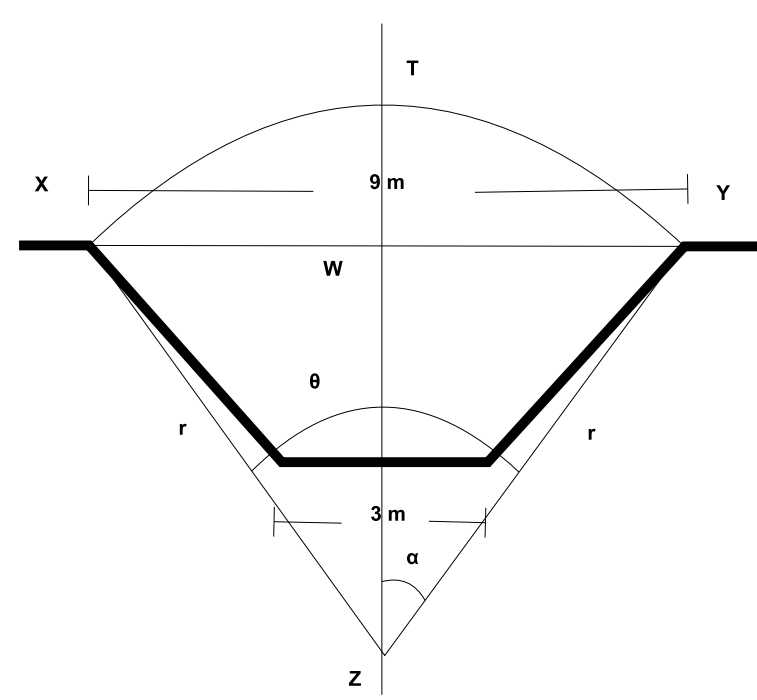

Figura 2. Cúpula del biodigestor.

Longitud del arco de la cúpula (la)

Es la trayectoria XTY en la Figura 2. Se calculó con la fórmula: la $=\theta \times \mathrm{r} \times \pi / 180 ; \mathrm{la}=9,93 \mathrm{~m}$

Apotema (ap) de la cúpula

Es el segmento WZ en la Figura 2. Se calculó con la fórmula:

$$
a p=\sqrt{r^{2}-\left(\frac{l p}{2}\right)^{2}}
$$

ap $=4,73 \mathrm{~m}$

Área del segmento circular (Aseg)

Aseg $=$ Área del sector circular $(X T Y Z)-$ Área del triángulo XYZ

$\operatorname{Aseg}=\left(\frac{1}{2} \times \frac{\pi}{1809} \times \theta \times \mathrm{r}^{2}\right)-\left(\frac{1 \mathrm{p} \times \mathrm{ap}}{2}\right)$

Aseg $=11,13 \mathrm{~m}^{2}$

Volumen de la cúpula del biodigestor (Vcb)

$V c b=$ Aseg $\times$ Largo del biodigestor

$\mathrm{Vcb}=133,56 \mathrm{~m}^{3}$

Capacidad de almacenamiento de biogás

Volumen libre en la cámara del biodigestor $=170 \mathrm{~m}^{3}-$ $126 \mathrm{~m}^{3}=44 \mathrm{~m}^{3}$

Volumen disponible para almacenar biogás en la cáma- ra y en la cúpula del biodigestor $=44 \mathrm{~m}^{3}+133,56 \mathrm{~m}^{3}=$ $177,56 \mathrm{~m}^{3}$

Volumen de biogás producido $=44,1 \mathrm{~m}^{3} /$ día

Capacidad de almacenamiento de biogás $=177,56$ $\mathrm{m}^{3} /\left(44,1 \mathrm{~m}^{3} /\right.$ día $)=4,03$ días

Si la mezcla estiércol: agua ocupara todo el volumen de la cámara del biodigestor $(\mathrm{V}=\mathrm{Vt})$, se producirán $59,5 \mathrm{~m}^{3}$ de biogás/día. Sólo quedará disponible el volumen de la cúpula para almacenar el biogás, lo que corresponderá a la producción de biogás de 2,24 días.

Área de geomembrana para el BTLC

Se calculó el área de geomembrana necesaria para la cámara del biodigestor, vereda, anclaje y cúpula.

Área lateral de la cámara del biodigestor: $\mathrm{AL}$

$$
A L=a p\left(\frac{P B+P b}{2}\right)+A b
$$

ap: apotema de la cámara del biodigestor

De la Figura 1:

$$
a p=\sqrt{3^{2}+((12-6) / 2)^{2}}
$$

$\mathrm{ap}=4,24 \mathrm{~m}$

PB: perímetro de la base mayor $=42 \mathrm{~m}$

$\mathrm{Pb}$ : perímetro de la base menor $=18 \mathrm{~m}$

$\mathrm{Ab}$ : área de la base menor $=18 \mathrm{~m}^{2}$

$\mathrm{AL}=145,2 \mathrm{~m}^{2}$

Área de la vereda: $\mathrm{AV}=2(0,5 \times 13)+2(9 \times 0,5)$ $=22 \mathrm{~m}^{2}$

Área sobre la viga de anclaje: $\mathrm{AA}=2(0,20 \times 13,4)+$ $2(0,2 \times 10,00) ; \mathrm{AA}=9,36 \mathrm{~m}^{2}$

Área de la cúpula: $\mathrm{ACU}=(\mathrm{la} \mathrm{x})+\mathrm{Aseg}+\mathrm{AV}+\mathrm{AA}$; $\mathrm{ACU}=161,65 \mathrm{~m}^{2}$

Área de geomembrana: Ageo $=\mathrm{AL}+\mathrm{AV}+\mathrm{AA}+\mathrm{ACU}$ ; Ageo $=338,21 \mathrm{~m}^{2}$

En consecuencia, se requieren 338,21 $\mathrm{m}^{2}$ de geomem- 
brana para recubrir la cámara del biodigestor y cubrir toda su superficie (cúpula) para tener un ambiente anaeróbico.

\section{Diseño de la laguna para almacenar biol}

En esta laguna se almacenará el efluente líquido del biodigestor (biol), producido durante diez días de operación, que representará el $80 \%$ del volumen total de esta laguna (VL). El efluente diario será igual al volumen de alimentación diaria del biodigestor (3,6 $\mathrm{m}^{3}$ de mezcla estiércol:agua). $\mathrm{VL}=45 \mathrm{~m}^{3}$

La laguna tendrá forma de tronco de pirámide invertida con una profundidad de 1,5 m (CNA, 2007) y un talud de $45^{\circ}$.

Empleando la ecuación (1) y de manera similar al cálculo de las dimensiones del BTLC, y considerando que el largo de la base menor será igual a dos veces su ancho:

Ancho de la base menor $=3,19 \mathrm{~m}$

Largo de la base menor $=6,38 \mathrm{~m}$

En consecuencia:

Ancho de la base mayor $=4,52 \mathrm{~m}$

Largo de la base mayor $=9,04 \mathrm{~m}$

Haciendo un reajuste en las dimensiones debido al talud de $45^{\circ}$ y a la profundidad de $1,5 \mathrm{~m}$, para llegar al volumen de $45 \mathrm{~m}^{3}$, se obtienen las dimensiones de la Figura 3.
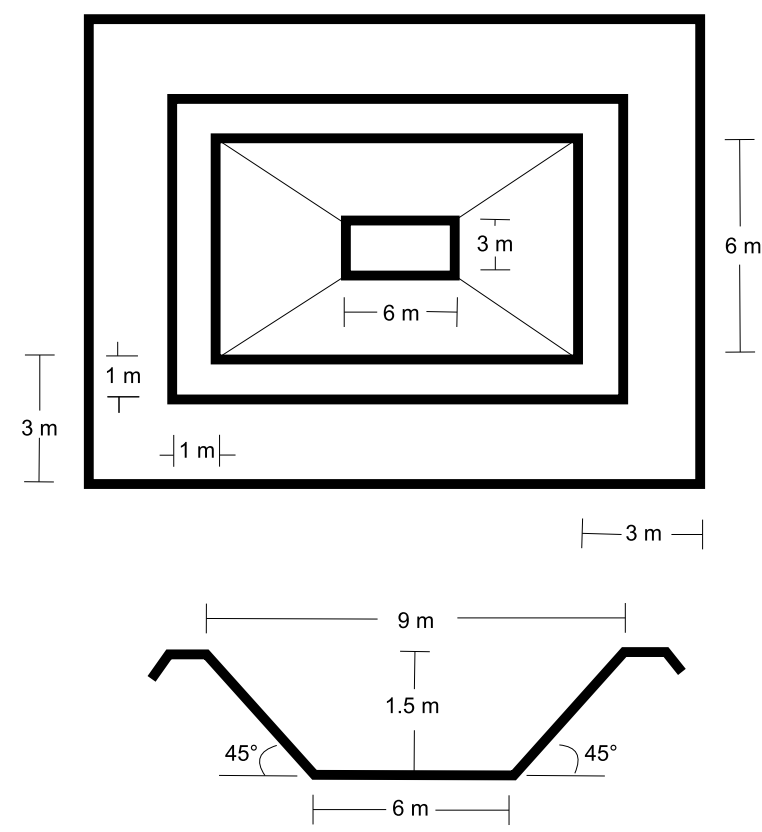

Figura 3. Vista superior y lateral de la laguna de almacenamiento de biol.
Base superior: $\operatorname{largo}=9 \mathrm{~m} ;$ ancho $=6 \mathrm{~m}$

Base inferior: $\operatorname{largo}=6 \mathrm{~m}$; ancho $=3 \mathrm{~m}$

Profundidad $=1,5 \mathrm{~m}$

Talud $=45^{\circ}$

Volumen $=51,6 \mathrm{~m}^{3}$

Esta laguna tendrá una corona de $3 \mathrm{~m}$ con una compactación del 85 al 90\% Proctor; una vereda de $1 \mathrm{~m}$ de ancho en todo su perímetro, iniciando en el borde de la laguna, cubierta con la misma geomembrana que impermeabilizará la laguna. A continuación de la vereda irá un metro más de geomembrana en todo el perímetro, la que se enterrará como anclaje.

Área lateral de la laguna: ALL

$$
A L L=a p L\left(\frac{P B L+P b L}{2}\right)+A b L
$$

Donde:

apL: apotema de la laguna:

$a p L=\sqrt{\left.1,5^{2}+(9-6) / 2\right)^{2}}=2,12 \mathrm{~m}$

PBL: perímetro de la base mayor de la laguna.

PbL: perímetro de la base menor de la laguna.

AbL: Área de la base menor de la laguna.

$\mathrm{ALL}=68,9 \mathrm{~m} 2$

Área de la vereda más anclaje: AVA

Vereda $=1 \mathrm{~m}$ en todo el perímetro.

Anclaje $=1 \mathrm{~m}$ en todo el perímetro.

$\mathrm{AVA}=2(13 \times 2)+2(6 \times 2)=76 \mathrm{~m} 2$

Área de geomembrana para la laguna: AGL

$$
\begin{gathered}
\mathrm{AGL}=\mathrm{ALL}+\mathrm{AVA} \\
\mathrm{AGL}=145 \mathrm{~m} 2
\end{gathered}
$$

Por lo tanto, para la laguna de almacenamiento de biol se requiere $145 \mathrm{~m} 2$ de geomembrana.

Diseño de la poza para secado de lodos de fondo (biosol)

Esta poza o cámara tiene dimensiones y forma simila- 
res a la cámara del biodigestor, pero de $2 \mathrm{~m}$ de profundidad, con una rampa para que pueda ingresar un cargador frontal para retirar los lodos frescos o secos, según se requiera para aplicar al campo como abono orgánico. Su volumen se calcula con la ecuación Vc, donde:

\section{$9 \mathrm{~m}$}

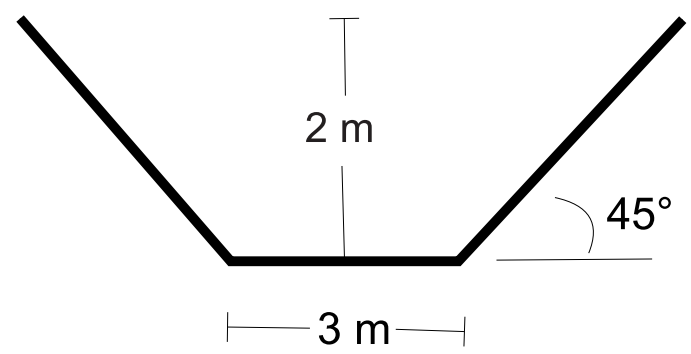

Figura 4. Dimensiones de la poza para secado de lodos de fondo.

\section{Diseño del sedimentador}

El sedimentador servirá para retirar los sólidos que vienen con el estiércol, los ligeros por flotación y los inertes (arena, tierra, piedras) por sedimentación. Será de concreto armado, con un área útil de $1,20 \mathrm{~m}^{2} \mathrm{y}$ un volumen de $1,00 \mathrm{~m}^{3}$; cuyas dimensiones se muestran en la Figura 5.

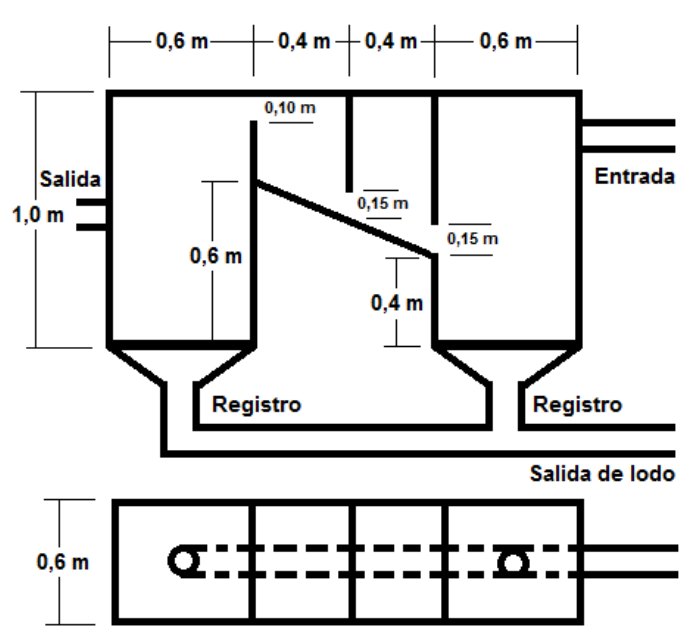

Figura 5. Dimensiones del sedimentador.

\section{IV.DISCUSIÓN}

La cantidad de biogás esperada de un digestor con una eficiencia razonable está relacionada con el contenido de sólidos volátiles. Funk (2007), menciona que el estiércol de una vaca lechera genera alrededor de 2,57 $\mathrm{m}^{3}$ de biogás. Pizarro et al. (2006), mencionan que la producción de biogás es alrededor de $2 \mathrm{~m}^{3} /$ vaca /día. En establos en México se ha reportado desde 1,4 a 2,08
$\mathrm{AB}$ : área de la base mayor $=9 \times 12=108 \mathrm{~m} 2$

$\mathrm{Ab}$ : área de la base menor $=3 \times 6=18 \mathrm{~m} 2$

h: profundidad de la cámara $=2 \mathrm{~m}$

$\mathrm{Vp}=113,39 \mathrm{~m} 3$
$12 \mathrm{~m}$

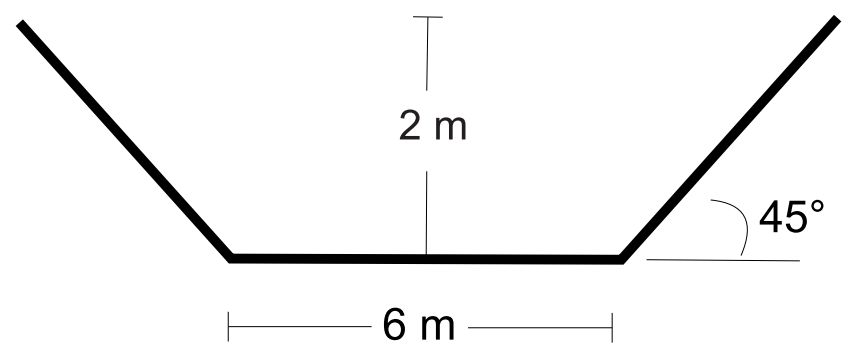

$\mathrm{m}^{3} / \mathrm{vaca} /$ día; la producción puede depender de las condiciones particulares de cada lugar y cada establo. Según el diseño realizado, a partir del estiércol de 40 vacas, del que se procesa $600 \mathrm{~kg} /$ día, el BTLC producirá $44,1 \mathrm{~m}^{3}$ de biogás/día, lo que equivale a $1,1 \mathrm{~m}^{3} \mathrm{de}$ biogás/vaca/día; que es un resultado adecuado.

La secuencia de diseño del biodigestor tipo laguna cubierta (BTLC) ha sido adaptada teniendo como base la empleada por Barrena et al. (2013), para biodigestores tubulares y por Guardado (2007), para biodigestores de cúpula fija tipo chino.

El biogás que se produzca en el BTLC que se ha diseñado para el Establo de la UNTRM llegará a un volumen de $44,1 \mathrm{~m}^{3} /$ día, y en el sistema se ha incluido y diseñado cartuchos rellenados con clavos de fierro de 2" para eliminar el $\mathrm{H}_{2} \mathrm{~S}$ del biogás de manera que cuando se lo emplee como combustible no cause corrosión en los componentes metálicos de los equipos; además, el volumen de biogás producido será suficiente para hacer funcionar un grupo electrógeno para abastecer con energía eléctrica al Establo de la UNTRM con lo que se reducirá su facturación por electricidad y se contribuirá a la sostenibilidad de su actividad. Asimismo, se empleará el biogás para calefacción de los terneros recién nacidos y para calentar agua para el lavado de las tuberías y accesorios del sistema de ordeño de las vacas.

La agitación del contenido del biodigestor con electro- 
bomba, recircula el biol hacia la entrada de la alimentación al BTLC, homogenizará su contenido y evitará que se forme costra en la superficie de la mezcla estiércol:agua en fermentación, que podría impedir el flujo del biogás hacia la cúpula del BTLC. Además, favorece la suspensión de sedimentos, distribuye los microorganismos que transforman el sustrato y reduce el tiempo de producción de biogás.

El BTLC procesará una gran cantidad de estiércol de ganado vacuno para producir biogás, cuyo principal componente es el metano con poder de GEI 21 veces mayor que el del $\mathrm{CO}_{2}$ (Moncayo, 2014); sin embargo, cuando se emplea el biogás como combustible, el metano se convierte en $\mathrm{CO}_{2}$, lo que reduce su poder de GEI y se protege al medio ambiente. Entonces, un sistema de producción de biogás y bioabonos reduce el impacto ambiental de la ganadería que según la Taramona et al. (2017), es una de las principales fuentes emisoras de GEI.

\section{CONCLUSIONES}

Se ha diseñado para la Estación Experimental Chachapoyas de la UNTRM, un biodigestor tipo laguna cubierta (BTLC) de $170 \mathrm{~m}^{3}$, con forma de tronco de pirámide invertida de $3 \mathrm{~m}$ de profundidad, con base inferior de $6 \mathrm{~m} \times 3 \mathrm{~m}$, base superior de $12 \mathrm{~m}$ x $9 \mathrm{~m}$ y talud de $45^{\circ}$, excavado en el suelo e impermeabilizado con geomembrana, donde se procesará el estiércol de 40 vacas que en promedio producen $600 \mathrm{~kg}$ de estiércol/día, en un volumen de trabajo de $126 \mathrm{~m}^{3}$ y tiempo de retención hidráulico de 35 días. La cúpula del biodigestor tendrá una altura de $1,8 \mathrm{~m}$ y $12 \mathrm{~m} \mathrm{x} 9 \mathrm{~m}$ en su base (borde superior del BTLC). Podrá almacenar 133,56 $\mathrm{m}^{3}$ de biogas. Entre el nivel de mezcla estiércol:agua contenido en el BTLC y su borde superior hay un volumen libre de $44 \mathrm{~m}^{3}$ para almacenar biogás, con lo que la capacidad de almacenamiento de biogás del BTLC será de $177,56 \mathrm{~m}^{3}$, que equivale a 4,03 días de producción de biogás.

La laguna para almacenar biol tiene forma de tronco de pirámide invertida impermeabilizada con geomembrana, de 1,5 $\mathrm{m}$ de profundidad, con base inferior de 6 $\mathrm{m} \times 3 \mathrm{~m}$, base superior de $9 \mathrm{~m} \times 6 \mathrm{~m}$, con talud de $45^{\circ}$, lo que hace un volumen total de $51,6 \mathrm{~m}^{3}$. La laguna para almacenar los lodos de fondo tiene forma de tronco de pirámide invertida, de 2,0 $\mathrm{m}$ de profundidad, con base inferior de $6 \mathrm{m \times} 3 \mathrm{~m}$, base superior de $12 \mathrm{mx} 9 \mathrm{~m}$, con talud de $45^{\circ}$ reforzado con canto rodado y cemento, lo que hace un volumen total de $113,39 \mathrm{~m}^{3}$.

\section{REFERENCIAS BIBLIOGRÁFICAS}

Barrena, M., L. Taramona, O. Gamarra, y M. Choy. 2013. Biodigestores tubulares para la producción de biogas. Lima (Perú): DAVISA E.I.R.L.

Bonilla, J. A. y C. Lemus. 2012. "Emisión de metano entérico por rumiantes y su contribución al calentamiento global y al cambio climático. Revisión." Revista Mexicana de Ciencias Pecuarias 3 (2): 215-246.

CNA(Comisión Nacional del Agua). 2007. Manual de agua potable, alcantarillado y saneamiento. Ciudad de México (México): SEMARNET y CONAGUA.

DEC (Dairy Environmental Committee). 2006. Dairying and the environment. Chicago (EE.UU.): DEC

Funk, T. 2007). Anaerobic methane digesters for dairy farms: Are you asking the right questions. Chicago(EE.UU.): University of Illinois.

Guardado, J. A. 2007. Diseño y construcción de plantas de biogás sencillas. Ciudad de la Habana (Cuba): CUBASOLAR

Laines, J. R. y Sosa, O. 2013. “Degradación anaerobia del contenido gástrico ruminal bovino para la obtención de biogás, en un biodigestor tipo cúpula." Ingeniería, Revista Académica de la FI-UADY 17 (1): 57-65.

Moncayo, G. 2014. Manual de dimensionamiento y diseño de biodigestores industriales para clima tropical. Biodigestores tropicalizados. Uelzen (Alemania): Aqualimpia Engineering. Nennich., T. D., J. H. Harrison, L. M. VanWleringen, D. Meyer, A. J. Heinrichs, W. P. Weiss, N. R. 
St-Pierre, R. L. Kincaid, D. L. Davidson, y E.

Block. 2005. "Prediction of manure and nutrient excretion fron dairy cattle." Journal of dairy science 88: 3721-3733.

Pizarro, C., W. Mulbry, D. Blersch, y P. Kangas. 2006. "An economic assessment of algal turf scrubber technology for treatment of dairy manure effluent." Journal of Ecological Engineering 26(4): 321-327.

SEMARNAT (Secretaría de Medio Ambiente y Recursos Naturales), SAGARPA (Secretaría de Agricultura, Ganadería, Desarrollo Rural, Pesca y Alimentación) y FIRCO (Fideicomiso de Riesgo Compartido). 2010. Especificaciones técnicas para el diseño y construcción de biodigestores en México. Ciudad de México (México): SEMARNAT, SAGARPA Y FIRCO.

Taramona, L. A., M. A. Barrena, O. A. Gamarra, M. Choy, y Y. Rodríguez. 2017. Rendimiento de biogás y sus beneficios socio económico en el desarrollo rural sostenible de las comunidades altoandinas. Ancash - Perú. Revista de Investigaciones de la Universidad Le Cordon Bleu 4 (2): 49-57. 